\title{
Spatiotemporal Scaling of Solar Surface Flows
}

\author{
J. K. Lawrence and A.C. Cadavid \\ Department of Physics and Astronomy, California State University, Northridge, California 91330-8268
}

A. Ruzmaikin

Jet Propulsion Laboratory, California Institute of Technology, Pasadena, California 91109

T. E. Berger

Lockheed Martin Space and Astrophysics Laboratory, Palo Alto, California 94304

(Received 22 March 2000; revised manuscript received 2 April 2001)

\begin{abstract}
The sun provides an excellent natural laboratory for nonlinear phenomena. We use motions of magnetic bright points on the solar surface, at the smallest scales yet observed, to study the small scale dynamics of the photospheric plasma. The paths of the bright points are analyzed within a continuous time random walk framework. Their spatial and temporal scaling suggests that the observed motions are the walks of imperfectly correlated tracers on a turbulent fluid flow in the lanes between granular convection cells.
\end{abstract}

DOI: $10.1103 /$ PhysRevLett.86.5894

In the outer $30 \%$ of its radius the sun's plasma is convectively unstable, and thermonuclear energy from the core is transported by overturning motion to the visible photosphere. The convective motions are most readily seen as the photospheric pattern of bright "granules" of hot, rising gas, surrounded by a network of "intergranular lanes" of cooler, darker, downflowing gas. The horizontal extent of a granular cell is $\sim 1000 \mathrm{~km}$ with a range of 200 to $2500 \mathrm{~km}$. The typical separation of granule centroids is $700 \mathrm{~km}$; typical lifetimes are 5-15 min.

Magnetic flux threading the solar surface is not uniformly distributed. By processes which are not well understood, it is concentrated into discrete kilogauss fibrils of diameter $\$ 150 \mathrm{~km}$. These accumulate in the intergranular lanes and vertices where they disturb the radiative properties of the photosphere and become apparent to observers through spectral line emission. Such magnetic bright points (MBPs) serve as tracers of the evolution of the granular flow field and of small scale motions in the intergranular network.

With Reynolds number $\approx 10^{7}$ turbulent flows are expected in the intergranular lanes. Numerical simulations of convection near the solar surface [1] indicate that turbulent vortices are formed in the intergranular lanes where the outflows from neighboring granules collide and turn downward. Observationally, spectrograms taken in magnetically and temperature insensitive spectral lines show line broadening in excess of known Doppler contributions, indicating the presence of turbulent flows on the borders of granules [2]. These observations cannot rule out other types of motion. However, the unique, high-resolution data set described below does provide access to the actual dynamics of the flows and reveals some of their quantitative characteristics.

Here we study subgranular motions at the highest resolution yet achieved [3-5]. The data were taken on 5 Oc-
PACS numbers: 47.27.-i, 05.40.Fb, 05.45.Df, 96.60.Mz

tober 1995 with the $50 \mathrm{~cm}$ Swedish Vacuum Solar Telescope on La Palma, Spain and consist of two cospatial and simultaneous (within $10 \mathrm{~ms}$ ) time series of 178 digital image pairs spanning $71 \mathrm{~min}$. One set of images was taken through a filter passing wavelengths $430.5 \pm 0.5 \mathrm{~nm}$ located in the $G$-band emission of the $\mathrm{CH}$ radical. The other set of images was made through a wide-band filter passing wavelengths $468.6 \pm 5.0 \mathrm{~nm}$ emphasizing the background continuum emission. A $21 \mathrm{Mm} \times 21 \mathrm{Mm}$ area of enhanced magnetic activity near the center of the solar disk was imaged, containing hundreds of MBPs. Exposure time was $20 \mathrm{~ms}$, and the pixel scale in the digital images was $60 \mathrm{~km}$ on the sun. The images were checked in real time for seeing sharpness, and the best image pair in each $25 \mathrm{~s}$ interval was recorded. Both time series were restored to near the telescope diffraction limit $(0.2 \mathrm{arc} \mathrm{sec})$ by phase diversity reconstruction [4]. The final resolution is $165 \mathrm{~km}$ in the $G$ band and $180 \mathrm{~km}$ in the broad-band channel. The images were further processed to remove instrumental effects and geometric distortion due to atmospheric seeing.

Next, the wide-band images were subtracted from their $G$-band counterparts, removing most of the granulation pattern common to both sets. Finally the images were subjected to a high-pass spatial filter to emphasize small scale features [6], and a threshold was imposed to identify MBPs. The result is a three-dimensional binary (MBP or not) array with two 350 pixel axes corresponding to $x$ and $y$ spatial directions and a third axis of 178 pixels corresponding to the time. A given MBP is tracked by segmentation of its binary object tree from the data cube. The segmentation is implemented using one-dimensional voxel addressing and searching over limited volumes around a given voxel for valid neighbors. After segmentation the individual tree is split into branches and the motion of each subobject is measured with respect to a common reference point. 


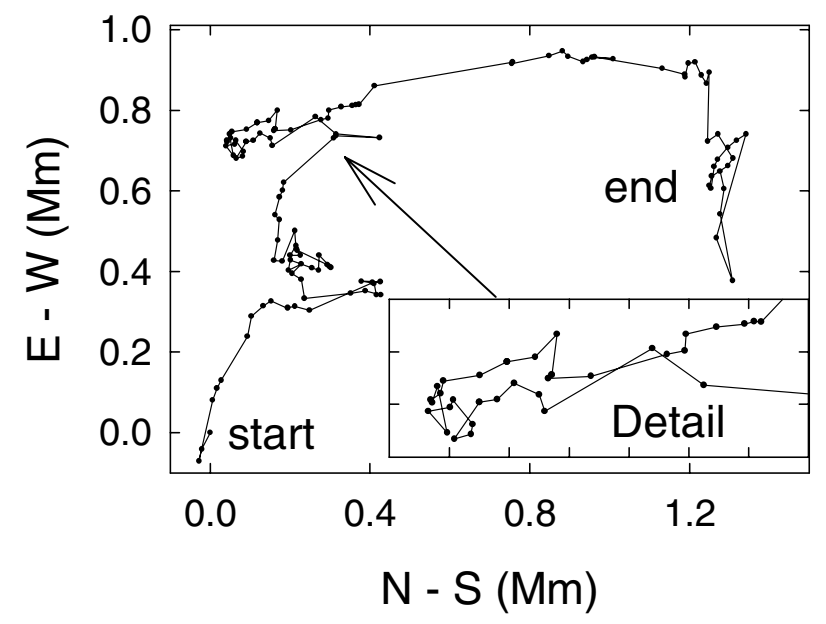

FIG. 1. Typical solar MBP trajectory. Distances are in megameters. Positions were observed at intervals of about $24 \mathrm{~s}$; the total elapsed time is $50 \mathrm{~min}$.

This procedure delivers strings of $x$ and $y$ positions and times, at roughly $24 \mathrm{~s}$ intervals, for 1800 individual MBPs. The longest sequence for an individual MBP object spans $71 \mathrm{~min}$, the shortest $9 \mathrm{~s}$. During approximately $1 \mathrm{~h}$ the MBPs typically cover an rms distance on the order of a granular diameter. Such an MBP path is illustrated in Fig. 1. The MBPs undergo significant morphological changes on a time scale of $100 \mathrm{~s}$ and are continuously merging and splitting as a result of their interaction with the granular flow [7]. Consequently, the data strings contain a number of large jumps, many representing artifacts related to the merging and splitting of two or more MBPs. For example, the trajectory shown in Fig. 1 contains four jumps with $v>c$; the largest is $v=14.6 \mathrm{~km}$. To ensure their removal, we follow past practice [3] and employ a cutoff above the photospheric sound speed $c=7 \mathrm{~km}$.

Because of the discrete structuring of the magnetic flux, the motions of the MBP tracers are naturally described in terms of random walks [8]. We will use a general equation for the time evolution of the $q$ th moment of the tracer displacements:

$$
\left\langle r^{q}\right\rangle^{1 / q}=D \cdot t^{\gamma(q) / 2},
$$

where $r$ is the total displacement during time $t$, and an appropriately weighted average is taken over all observed displacements. Figure 2 shows $\left\langle r^{q}\right\rangle^{1 / q}$ as a function of $t$ for two values of $q$. We see excellent power law scaling over 2 orders of magnitude, up to $35 \mathrm{~min}$ in time. This permits precise determinations of $\gamma(q)$, which are shown in Fig. 3 . While Brownian walks give $\gamma(q)=1$ for all $q>-1$, we find that $\gamma(q)>1$ and varies with $q$. Although diffusion studies are usually limited to mean square displacements, i.e., $q=2$, here the general $q$ dependence proves to be crucial for correct analysis of the motions.

Figures 2 and 3 indicate that for the MBP motions $\gamma(2)=1.13 \pm 0.01$ implying superdiffusion, and that in the limit of small $q$ we approach the even more

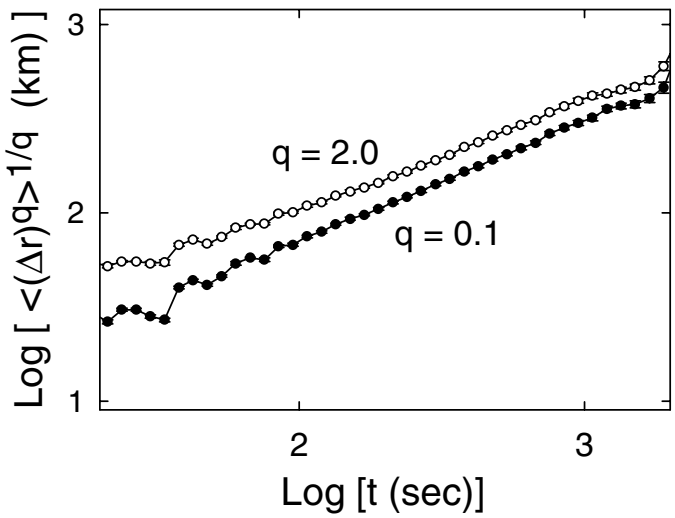

FIG. 2. Moments $\left\langle r^{q}\right\rangle^{1 / q}$ of the cumulative solar MBP displacements $r$ in megameters versus time in minutes for a sonic velocity cutoff and moment orders $q=0.1$ (filled circles) and $q=2$ (open circles). If error bars are not visible, they are smaller than the symbols.

superdiffusive value $\gamma(0)=1.27 \pm 0.01$. Our analysis below will indicate that in the ideal situation of infinite, precise data we should find that $\gamma(q)$ is independent of $q$. The problem here is to find that ideal value from limited, imperfect data. When we use no velocity cutoff, a case not illustrated, we find $\gamma(2)<1$ indicating subdiffusion, but again that $\gamma(0) \approx 1.3$. We will see below that it is $\gamma(0)$, rather than $\gamma(2)$, that reliably characterizes the MBP walks.

The superdiffusion we have found fits naturally into the framework of the continuous time random walk (CTRW) approach [9]. This method focuses on the statistical properties of the processes under consideration. It handles superdiffusion by means of Lévy flights, with an infinite mean squared displacement, by adding a time cost to the long jumps, so that the mean squared displacement as a function of time converges. In a broader context, previous experimental work [10] has shown that tracer motion in a

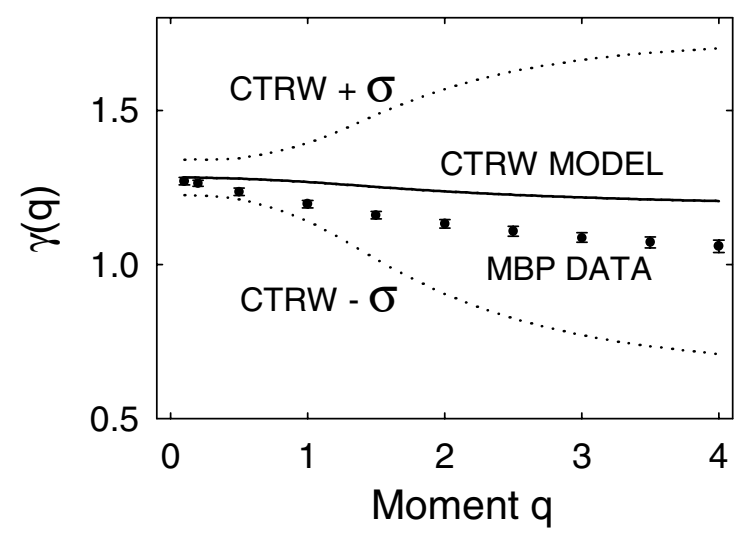

FIG. 3. The multiscaling exponent $\gamma(q)$ versus moment order $q$. Solid line: solar MBPs with a sonic cutoff $v \leq c$; open circles: continuous time random walk (CTRW) mean value for 1000 runs of 100 walks each; dotted lines: 1 standard deviation limits of the CTRW case indicating the expected range for a single 100 walk realization. 


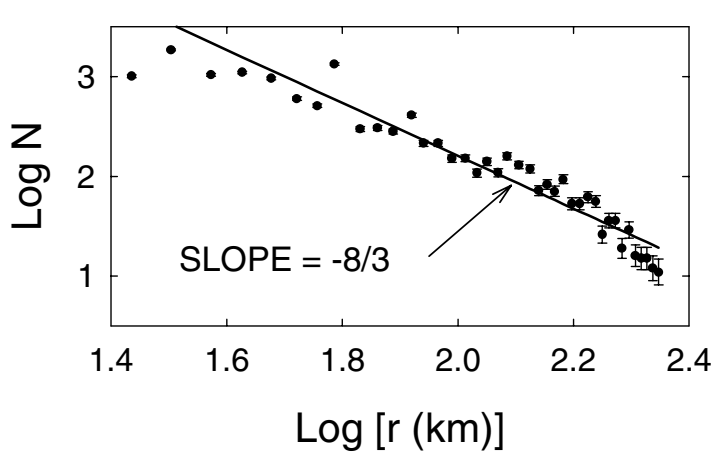

FIG. 4. Filled circles: Logarithms of numbers of step lengths in $5 \mathrm{~km}$ bins versus the logarithm of step lengths. The solid line has a slope of $-8 / 3$ and represents a fit for all $r>40 \mathrm{~km}$.

rotating flow presents anomalous diffusion which can be analyzed in terms of Lévy flight motions as we do here. More recent experimental work [11] finds that the separations of particle pairs in a turbulent flow, in the Richardson regime, show a stretched exponential form rather than the Lévy power law form. This result, however, can be recovered from particle pairs undergoing relative motions with an asymmetric Lévy distribution [12].

Our use of the CTRW technique is based on two adjustable parameters. The first, $\lambda$, is the exponent of a power law distribution $\sim r^{-\lambda}$ of spatial steps of tracers due to underlying fluid motions. The second, $\nu$, connects the spatial step lengths $r$ to elapsed times according to $r \propto t^{\nu}$. By looking at the spatial scaling properties of the tracer motions, we can directly estimate these parameters for our case. Figure 4 shows the distribution of lengths for all solar MBP steps between consecutive images excluding those with $v>c$. A fit for all $r \geq 40 \mathrm{~km}$ gives $\lambda=2.65 \pm 0.13 \approx 8 / 3$. The assumed step time scaling gives a velocity $v=r / t \propto r^{1-1 / \nu}$. We estimate this exponent by calculating the velocities of all solar MBP steps with $t \leq 1000 \mathrm{~s}$, but rejecting those with $v>c$. A plot of $v$ versus $r$ is shown in Fig. 5. The best fit for $20 \leq r \leq$ $1000 \mathrm{~km}$ gives $1-1 / \nu=0.35 \pm 0.03 \approx 1 / 3$. Thus we

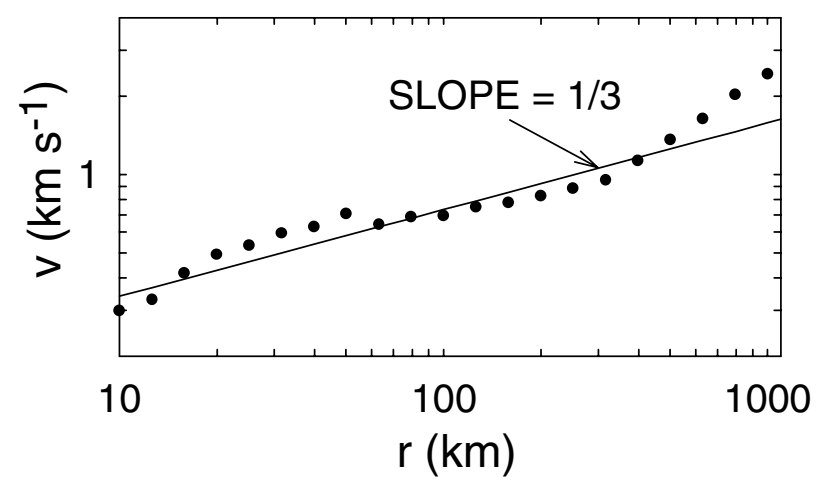

FIG. 5. Filled circles: Step velocities of MBP motions versus step length for all steps occurring over $1000 \mathrm{~s}$ or less and with $v \leq 7 \mathrm{~km}$. Error bars are smaller than the symbols. Solid line: best linear fit for step lengths between 20 and $1000 \mathrm{~km}$. The slope is $0.35 \pm 0.03 \approx 1 / 3$. find $v \propto r^{1 / 3}$, leading to $\nu \approx 1.5 \pm 0.2$ and implying Kolmogorov "K41" scaling with $r^{2} \propto t^{3}[13,14]$. However, we have found above that the MBP random walks give $\left\langle r^{2}\right\rangle \propto t^{\gamma}$ with $\gamma$ superdiffusive but less than 3. A key result of the CTRW approach is that, because the tracers may be imperfectly correlated with the underlying fluid flow, "Kolmogorov scaling does not necessarily imply Richardson's law [15]."

To connect our notation with that common in the CTRW literature $[9,15,16]$ we note that the formalism is based on a probability density function $\psi(r, t)=C r^{-\mu} \delta\left(r-t^{\nu}\right)$. Our parameter $\lambda=\mu-1 / \nu-(d-2)$. In the present case the dimension $d=2$. The $1 / \nu$ term arises from the measure associated with the delta function argument.

The CTRW technique allows analytical calculation of $\left\langle r^{q}\right\rangle^{1 / q} \propto t^{\gamma(q) / 2}$ for $q=2$. The time evolution of the moments, that is, the value of $\gamma$, is strongly dependent on the values of $\lambda$ and $\nu$. There are four different cases depending on the degree to which the tracers we follow are coupled to the fluid motions. These are illustrated in Fig. 6 as regions in the two-dimensional $\lambda, \nu$ plane. In case I with $\lambda \geq 3$ and $\nu \geq 1 /(\lambda-1)$ one finds $\gamma(2)=1$. This corresponds to a brief correlation time $\tau$ of the tracers with the flow and thus normal Brownian diffusion. In case II $\lambda>3$ but $\nu<1 /(\lambda-1)$. This corresponds to strong coupling with $\tau=\infty$ and $\gamma(2)=\nu(\lambda-1)<1$ giving subdiffusion. In case III $\lambda<3$ and $\nu>1 /(\lambda-1)$. In this intermediate case $\tau$ is finite but includes significant coupling of the tracers with the flow. We find

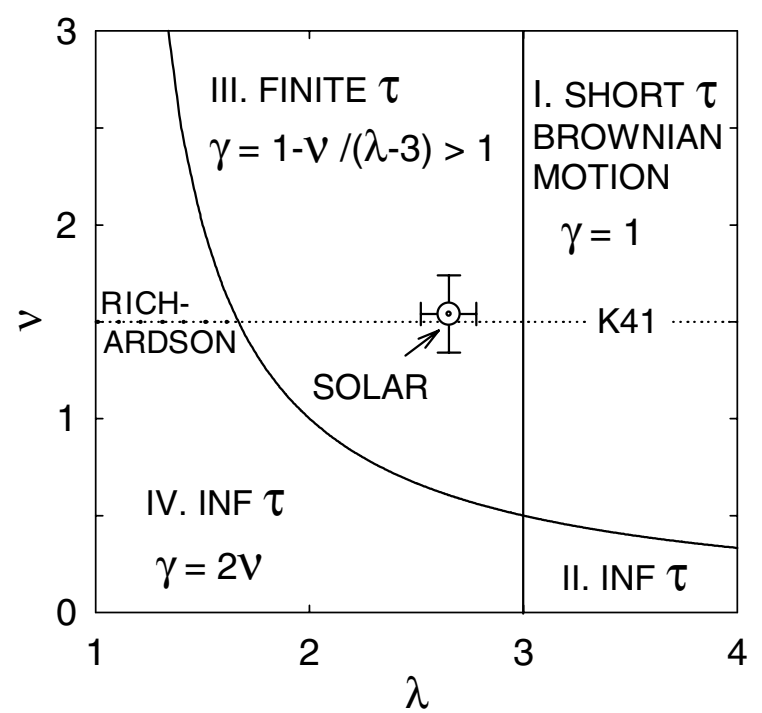

FIG. 6. The two-dimensional $\lambda, \nu$ parameter space. The solid lines $\lambda=3$ and $\nu=1 /(\lambda-3)$ divide the space into four regions with differing correlation properties of the tracers with the underlying flows and differing diffusion exponents. The dotted line at $\nu=3 / 2$ indicates Kolmogorov scaling. The heavier dotted line at $\nu=3 / 2$ and $\lambda<5 / 3$ indicates the locus of Richardson diffusion with $\gamma=3$. The solar symbol represents the values of $\lambda$ and $\mu$ estimated directly from the solar MBP walks. 
$\gamma(2)=1-\nu(\lambda-3)>1$ and superdiffusion. Finally, in case IV we have $1 \leq \lambda<3$ and $\nu \leq 1 /(\lambda-1)$. Now $\tau=\infty$, and the tracers are completely coupled to the flow. We find $\gamma(2)=2 \nu$. This is superdiffusive when $\nu>1 / 2$.

The analytical calculation of $\gamma(2)$ is verified by numerical simulation [16]. We extend the simulations to arbitrary order in the range $0.1 \leq q \leq 4.0$ by adopting a simple, repetitive algorithm for generalized random walks: step in a random direction by a distance $r$ chosen with a probability $\propto r^{-\lambda}$ while advancing the time by $t \propto r^{1 / \nu}$.

In the limit of many trials we find that $\gamma(q) \rightarrow \gamma=$ const, which differs from the decreasing $\gamma(q)$ shown by the solar MBPs in Fig. 3. However, the data set is limited in time and number of tracers so the statistics are important. Let $\tilde{\gamma}(q)$ represent the diffusion exponent calculated by averaging over 100 walks simulated with our CTRW algorithm, roughly matching the observational situation. An average over 1000 such $\tilde{\gamma}(q)$ gives a constant $\langle\tilde{\gamma}(q)\rangle \approx 1.3$, as illustrated in Fig. 3 for a simulation with $\lambda=8 / 3$ chosen to match the solar data. Also shown in Fig. 3 are the 1 standard deviation limits of the 1000 runs. These indicate the range within which one expects to find a single realization of $\tilde{\gamma}(q)$. Unless $q \ll 1$, the form of an individual $\tilde{\gamma}(q)$ is dominated by a small number of the largest jumps. Depending on their timing in individual runs they can give large fluctuations in, for example, $\tilde{\gamma}(2)$. For $q \ll 1$ the values of $\tilde{\gamma}(q)$ are determined more equally by all jumps and characterize the asymptotic mean of $\gamma(q)$ for all $q$. The distribution of the underlying $\tilde{\gamma}(q)$ is narrow for $q \lessgtr 0.5$ permitting a robust measurement.

The values of $\lambda$ and $\nu$ determined from the scaling of the solar MBP space and time steps are shown on the plot of Fig. 6. These place the solar flows on the $\nu=3 / 2$ line corresponding to Kolmogorov scaling. However, the value of $\lambda$ selects the superdiffusive intermediate regime between Brownian and Richardson diffusion. Thus, the MBPs are imperfect tracers of the flow. For the Richardson case with $\gamma=3$ we would need perfect correlation of the MBPs with the flow. Instead the parameters predict a diffusion exponent $\gamma=1.54 \pm 0.39$. From the simulations based on the same $\lambda$ and $\nu$ we find $\gamma(0.1)=1.28 \pm 0.05$, and $\gamma(2)=1.24 \pm 0.33$; the uncertainties show why using $q \rightarrow 0$ is preferable to using $q=2$ when dealing with data. By direct measurement of the random walks we found $\gamma(0)=1.27 \pm 0.01$ and $\gamma(2)=1.13 \pm 0.01$. Mindful of the limited data set, we adopt the larger value. The result $\gamma \approx 1.3$ holds independent of any velocity cutoff.

It is no surprise that the MBPs are imperfect tracers of the fluid flows. They are not truly local objects, but rather the intersections with the photosphere of extended flux tubes which are influenced by various motions at any given time and are restricted to intergranular lanes. Nevertheless, the CTRW approach to anomalous diffusion via Lévy flights reveals quantitative properties of the small scale photospheric flows and their coupling to the magnetic flux tubes. The spatiotemporal scaling of the MBP dynamics indeed indicates the presence of turbulence in intergranular lanes. Our quantifying of the deviation of the MBP motions from the underlying fluid motion should contribute to a better physical understanding of flux tubes and the solar medium.

This work was supported in part by NSF Grants No. ATM-9628882 and No. ATM-9987305. T.E. B. was supported by NASA SR\&T Contract No. NASW-98008. The SVST is operated by the Swedish Royal Academy of Sciences at the Spanish Observatorio del Roque de los Muchachos of the Instituto de Astrofisica de Canarias.

[1] R. F. Stein and A. Nordlund, Astrophys. J. 499, 914 (1998).

[2] A. Nesis, A. Hanslmeier, R. Hammer, R. Komm, W. Mattig, and J. Staiger, Astron. Astrophys. 279, 599 (1993).

[3] T.E. Berger and M. G. Löfdahl, R. A. Shine, and A. M. Title, Astrophys. J. 506, 439 (1998).

[4] M. G. Löfdahl, T. E. Berger, R. A. Shine, and A. M. Title, Astrophys. J. 495, 965 (1998).

[5] T.E. Berger, M. G. Löfdahl, R. A. Shine, and A. M. Title, Astrophys. J. 495, 973 (1998).

[6] T.E. Berger, C. J. Schrijver, R. A. Shine, T. D. Tarbell, A. M. Title, and G. Scharmer, Astrophys. J. 454, 531 (1995).

[7] T.E. Berger and A. M. Title, Astrophys. J. 463, 797 (1996).

[8] R. B. Leighton, Astrophys. J. 140, 249 (1964).

[9] J. Klafter, A. Blumen, and M. F. Shlesinger, Phys. Rev. A 35, 3081 (1987).

[10] T. H. Solomon, E. R. Weeks, and H. L. Swinney, Phys. Rev. Lett. 71, 3975 (1993).

[11] M.-C. Jullien, J. Paret, and P. Tabeling, Phys. Rev. Lett. 82, 2872 (1999).

[12] I. M. Sokolov, J. Klafter, and A. Blumen, Phys. Rev. E 61, 2717 (2000).

[13] A. N. Kolmogorov, Dokl. Akad. Nauk (USSR) 30, 301 (1941) [reprinted in Proc. R. Soc. London A 434, 9 (1991)].

[14] U. Frisch, Turbulence: The Legacy of A. N. Kolmogorov (Cambridge University Press, Cambridge, 1995).

[15] M. F. Shlesinger, B. J. West, and J. Klafter, Phys. Rev. Lett. 58, 1100 (1987).

[16] G. Zumofen, A. Blumen, J. Klafter, and M. F. Shlesinger, J. Stat. Phys. 54, 1519 (1989). 